L'importance de posséder des données complètes et actuelles est évidente pour gérer le système de santé et nous avons besoins des nôtres pour participer activement à cette gestion! Or, la statistique des médecins avait tranquillement vieilli et ne correspondait plus aux besoins, ni surtout à la réalité du terrain, au mode de travail actuel des médecins.

C'est donc à une réforme en profondeur de cette statistique des médecins que s'est attelée la division
«Données, démographie et qualité» du Secrétariat général de la $\mathrm{FMH}$, sous la direction des deux auteures de l'éditorial de ce jour. A sa lecture, vous comprendrez mieux de quoi il retourne et apprécierez l'importance du travail fourni - merci à elles!

Jacques de Haller, président de la FMH

\title{
Connaître les données pour planifier efficacement
}

De combien de médecins la Suisse a-t-elle besoin et aura-telle besoin à l'avenir? Indépendamment de la décision du Parlement sur la clause du besoin, cette question reste d'actualité. Quant à une régulation des prestataires de soins adaptée aux besoins, elle demeure aussi à l'ordre du jour. De l'avis de la FMH, une pratique convaincante de l'admission des médecins à pratiquer doit satisfaire aux critères suivants: elle tient compte de chaque situation cantonale et régionale en matière de couverture médicale et de besoins en soins, elle porte sur les secteurs tant ambulatoire que semi-hospitalier et hospitalier, et elle est gérée par les cantons, qui intègrent systématiquement les organes concernés du corps médical dans la procédure d'admission.

Bien sûr, une régulation convaincante et conforme aux besoins doit aussi se fonder sur des données complètes et fiables. La FMH suit et analyse depuis longtemps l'évolution du corps médical exerçant en Suisse - les données qu'elle recense à ce sujet sont analysées dans la statistique médicale de la FMH et publiées sur www.fmh.ch.

En démographie médicale, certains facteurs qui n'étaient pas représentés jusqu'ici dans la statistique de la FMH, ou alors de façon trop peu différenciée, ont gagné en importance ces derniers temps: par exemple le recen- sement du travail à temps partiel. Pour pouvoir répondre aux questions qui se posent aujourd'hui et se poseront dans le futur, la FMH est en train de réviser sa statistique médicale. D'ici environ la fin de l'année, celle-ci permettra, outre la saisie de données concernant la sociodémographie et les qualifications des médecins (formation pré-, postgraduée et continue), de tirer des conclusions sur l'activité du corps médical suisse. L'article publié à la page suivante de ce numéro commente en détail cette révision de la statistique médicale de la FMH.

En élargissant sa statistique médicale, la FMH fournit une contribution et des arguments essentiels à une discussion approfondie sur une régulation du corps médical fondée sur les besoins. A moyen terme, un monitorage de l'ensemble des prestataires, tous secteurs confondus, sera nécessaire afin de disposer de données fiables pour gérer la fourniture de soins - la statistique médicale de la FMH pourrait devenir un élément de ce processus!

Pour la direction du projet de révision de la statistique médicale de la FMH:

Martina Hersperger, responsable du département $D D Q$

Esther Kraft, collaboratrice scientifique du département DDQ 